\title{
Evolution of young brown dwarf disks in the mid-infrared
}

\author{
M. F. Sterzik ${ }^{1}$, I. Pascucci ${ }^{2}$, D. Apai ${ }^{2}$, N. van der Bliek ${ }^{3}$, and C. P. Dullemond ${ }^{4}$ \\ 1 European Southern Observatory, Casilla 19001, Santiago 19, Chile \\ e-mail: msterzik@eso.org \\ 2 Max-Planck Institute für Astronomie, Königstuhl 17, 69117 Heidelberg, Germany \\ 3 Cerro Tololo Inter-American Observatory, Casilla 603, La Serena, Chile \\ 4 Max-Planck Institute für Astrophysik, Postfach 1317, 85748 Garching bei München, Germany
}

Received 21 June 2004 / Accepted 30 August 2004

\begin{abstract}
We have imaged two bona-fide brown dwarfs with TReCS/GEMINI-S and find mid-infrared excess emission that can be explained by optically thick dust disk models. In the case of the young $(\approx 2 \mathrm{Myr})$ Cha $\mathrm{H} \alpha 1$ we measure fluxes at $10.4 \mu \mathrm{m}$ and $12.3 \mu \mathrm{m}$ that are fully consistent with a standard flared disk model and prominent silicate emission. For the $\approx 10 \mathrm{Myr}$ old brown dwarf 2MASS1207-3932 located in the TW Hydrae association we find excess emission at $8.7 \mu \mathrm{m}$ and $10.4 \mu \mathrm{m}$ with respect to the photosphere, and confirm disk accretion as a likely cause of its strong activity. Disks around brown dwarfs likely last at least as long as their low-mass stellar counterparts in the T-Tauri phase. Grain growth, dust settling, and evolution of the geometry of brown dwarf disks may appear on a timescale of $10 \mathrm{Myr}$ and can be witnessed by observations in the mid-infrared.
\end{abstract}

Key words. accretion, accretion disks - stars: low-mass, brown dwarfs - stars: formation

\section{Introduction}

There is mounting evidence that dusty disks accompany brown dwarfs (BDs) in their early evolutionary stages similar to circumstellar disks around T Tauri stars (TTS). Based on excess emission in the near-infrared $(J H K)$, Muench et al. (2001) infer that many substellar objects are initially surrounded by circumstellar disks. At longer wavelengths the effects of disk emission become more pronounced compared to the (cool) brown dwarf photosphere. Jayawardhana et al. (2003) and Liu et al. (2003) find $L^{\prime}(3.8 \mu \mathrm{m})$ excess emission in the majority of substellar objects in young clusters. The frequency of young circumstellar disks appears to be similar in the stellar and in the substellar regime. Physical properties of dust disks around BDs can be better determined with mid-infrared (MIR) observations. Exploiting the ISOCAM surveys at $6.7 \mu \mathrm{m}$ and $14.3 \mu \mathrm{m}$ in Chameleon (Persi et al. 2000) and $\rho$-Oph (Bontemps et al. 2001), Natta \& Testi (2001) and Natta et al. (2002) tried to explain the spectral energy distribution (SED) of the associated young BD population. They show that the MIR excess emission of these objects is generally consistent with predictions of simple, passive disk models. This suggests far-reaching implications such as that the general formation mechanism of stars by core collapse and formation of an accretion disk extends into the sub-stellar regime.

Important physical properties of sub-stellar disks remain to be understood. For example, the assumption of hydrostatic equilibrium in the vertical disk direction - reasonably accurate for many $\mathrm{T}$ Tauri disks - implies a flared geometry, and the presence of an optically thin surface layer that is heated by direct illumination by the central source (Chiang \& Goldreich 1997; Chiang et al. 2001; Natta et al. 2001). In these upper layers a prominent emission signature of silicates is expected around $10 \mu \mathrm{m}$, as observed for many disks around Herbig Ae/Be stars (Meeus et al. 2001) and TTS (Meeus et al. 2003; Pryzgodda et al. 2003). However, the majority of disk models that actually fit SEDs of young BDs apparently require a flat geometry (Natta et al. 2002). Alternatively, or in addition to geometrical effects, grain growth can weaken, or even extinguish the $10 \mu \mathrm{m}$ signature (Bouwman et al. 2001). Unfortunately, the poor accuracy and wavelength sampling of the ISO photometry make it difficult to draw unambiguous conclusions.

New, sensitive, mid-infrared instruments at large telescopes allow us to probe the $10 \mu \mathrm{m}$ wavelength range of the SEDs of young BDs in much greater detail and with higher accuracy. The first ground-based MIR detection of a disk around the young $\mathrm{BD}$ candidate $\mathrm{ChaH} \alpha 2$ unequivocally demonstrates the absence of a silicate emission feature in its disk (Apai et al. 2002). Both, flared and flat disk models are able to explain the SED of three young brown dwarfs GY5, GY11 and GY310 (Mohanty et al. 2004). Apai et al. (2004) present first evidence for grain growth and dust settling within the disk of the young brown dwarf CFHT BD Tau 4, the best characterized BD disk up to date (Pascucci et al. 2003; Klein et al. 2003).

In this paper we report high-quality MIR photometry of two bona-fide BDs, namely ChaH $\alpha 1$ and 2MASS1207-3932 (in the following 2M1207), obtained with the Thermal-Region Camera Spectrograph T-ReCS mounted on Gemini-South. 


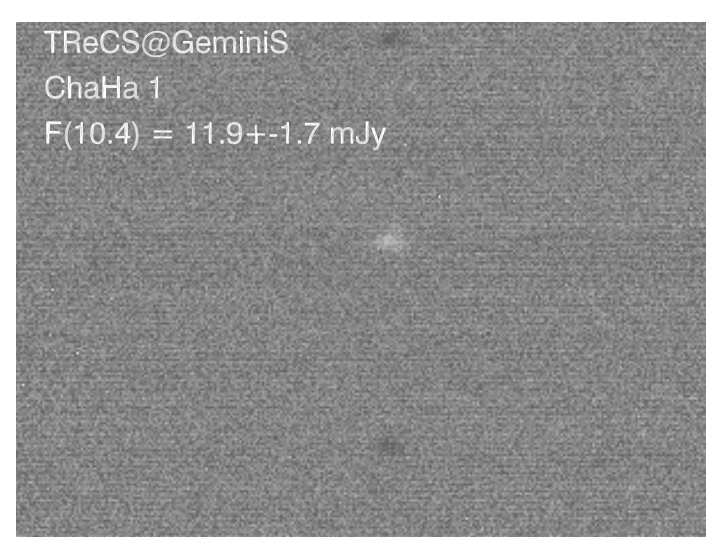

Fig. 1. TReCS image of ChaH $\alpha 1$ at $10.4 \mu \mathrm{m}$. Note the smearing due to imperfect tracking.

A disk around ChaH $\alpha 1$ has already been inferred from ISOCAM observations (Natta \& Testi 2001). With the help of our new photometric values we refine the model for its disk. For 2M1207 we present the first direct evidence for the existence of circum(sub)stellar material around an approximately 10 Myr old object. 2M1207 has been identified as BD by optical spectroscopy (Gizis 2002), and is related to the TW Hydrae association (TWA), a loose group of $\sim 10$ Myr old T Tauri stars at an approximate distance of $60 \mathrm{pc}$ to the sun (Webb et al. 1999). The lack of a measured $K-L^{\prime}$ excess did not indicate the presence of any circum-sub-stellar material (Jayawardhana et al. 2003), but strong emission in $\mathrm{H} \alpha$ and HeI suggested indirectly that this object may be a (weak) accretor (Mohanty et al. 2003; in the following MJBN). Our new measurements - to our knowledge among the most sensitive ground-based mid-infrared photometry ever reported - demonstrate the persistence of dust around older BDs, on timescales at least as long as for low-mass stars.

\section{Observations and data reduction}

The imaging observations were performed in service mode with TReCS (Telesco et al. 1998) mounted on GeminiSouth on Jan. 2, $2004(\mathrm{ChaH} \alpha 1)$ and on Jan. 27, 2004 (2MASS1207-3932) under clear weather conditions. Its pixel scale of $0.09^{\prime \prime}$ results in a field of view of $\approx 29^{\prime \prime} \times 22^{\prime \prime}$. The same throw amplitudes, but opposite directions, were used for chopping and nodding. The observations on Jan. 2 were obtained with throw amplitudes of $10^{\prime \prime}$, and the positive and two negative beams are all located in the field of view. The source appears only in the final, co-added frame, because slight tracking errors of the telescope introduced some image smearing of about 2-3 pixels. A more accurate correction of the tracking errors was not possible due to the faintness of ChaH $\alpha 1$, and "shift and add" co-adding of individual chopping pairs was not possible (in contrast to the case of BD-Tau4 discussed in Apai et al. 2004). Figure 1 shows the final frame with the detection. $2 \mathrm{M} 1207$ was observed with $15^{\prime \prime}$ throw amplitudes, and only the overlay of the two positive beams can be extracted in the final image. No tracking errors were evident, and the target appears point-like in the final image, (Fig. 2). A summary of the observing log, including total integration times, is given in Table 1.

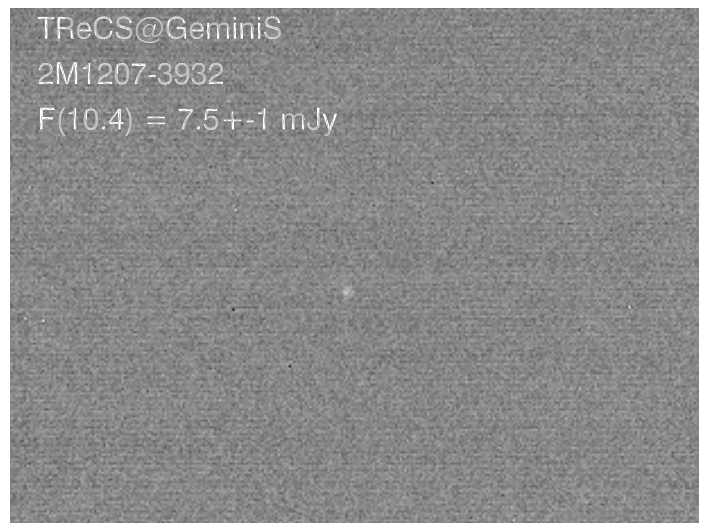

Fig. 2. TReCS image of 2M1207-3932 at 10.4 $\mu \mathrm{m}$. Chop and nod amplitudes were larger than the field-of view $\left(\approx 29^{\prime \prime} \times 22^{\prime \prime}\right)$.

Table 1. Log of the TReCS observations.

\begin{tabular}{llllll}
\hline \hline RJD & Object & Filter & Airmass & Int[s] & Calibrator \\
\hline 53006.74 & ChaH $\alpha 1$ & Si1-7.7 & 1.6 & 782 & HD 92305 \\
53006.70 & ChaH $\alpha 1$ & Si4-10.4 & 1.6 & 912 & HD 92305 \\
53006.79 & $\mathrm{ChaH} \alpha 1$ & Si6-12.3 & 1.5 & 1216 & HD 92305 \\
53031.84 & $2 \mathrm{M} 1207$ & Si1-7.7 & 1.0 & 304 & HD 110458 \\
53031.85 & 2M1207 & Si2-8.7 & 1.0 & 608 & HD 110458 \\
53031.81 & 2M1207 & Si4-10.4 & 1.0 & 782 & HD 110458 \\
\hline
\end{tabular}

Final co-added images were obtained from TReCS pipeline processed images and from manual merging of individual frames, with consistent results. Source count-rates were extracted with standard aperture photometry. Curve-of-growth methods were applied to find the aperture radius that maximized the signal-to-noise ratio of the extracted source. That radius was then used for the calibration source to determine the count-rate to flux conversion factor. Error estimates are based on the formal error of the background noise weighted over the source extraction region. When positive and negative beams were available, the fluxes were found to be consistent with these formal error estimates. The stellar parameters assumed, and mid-infrared fluxes observed are summarized in Table 2.

Given the effective temperatures and bolometric luminosities indicated in Table 2, the purely photospheric contribution to the $10.4 \mu \mathrm{m}$ flux density is about $1 \mathrm{mJy}$ for $\mathrm{ChaH} \alpha 1$ and $1.5 \mathrm{mJy}$ for $2 \mathrm{M} 1207$. This estimate is based on the assumed distances (see Sects. 3.1 and 3.2) and on BD atmospheric models from the Lyon group (e.g. Allard et al. 2000, 2001). In the following, we use their published tables that include full dust treatment (both condensation and opacities) and use the $\mathrm{TiO}$ and $\mathrm{H}_{2} \mathrm{O}$ line lists from Nasa AMES. We refer to a surface gravity of $\log (g)=3.5$ and solar metallicity. The atmospheric fluxes in the N-band in these models do not vary by more than $1 \%$ from a blackbody flux assuming the same $T_{\text {eff }}$ (in contrast to the near-IR, where the deviations can be huge). Hence, both our measurements indicate significant excess that cannot come from a BD photosphere alone. An excess criterium based only on NIR photometry (like $E\left(K-L^{\prime}\right)_{0}$ used e.g. in Jayawardhana et al. 2003) has very limited value for signalling the presence 
Table 2. Stellar parameters and mid-infrared fluxes. $7.7 \mu \mathrm{m}$ upper limits, and $8.7 \mu \mathrm{m}, 10.4 \mu \mathrm{m}, 12.3 \mu \mathrm{m}$ measurements are from this work.

\begin{tabular}{lllllllllllll}
\hline \hline Object & SpT & $\begin{array}{l}T_{\text {eff }} \\
{[\mathrm{K}]}\end{array}$ & $\begin{array}{l}L_{\text {bol }} \\
{\left[L_{\odot}\right]}\end{array}$ & $\begin{array}{l}M \\
{\left[M_{\odot}\right]}\end{array}$ & $\begin{array}{l}\text { Age } \\
{[\mathrm{Myr}]}\end{array}$ & $\begin{array}{l}3.8 \mu m^{a} \\
{[\mathrm{mJy}]}\end{array}$ & $\begin{array}{l}6.7 \mu \mathrm{m} \\
{[\mathrm{mJy}]}\end{array}$ & $\begin{array}{l}7.7 \mu m^{d} \\
{[\mathrm{mJy}]}\end{array}$ & $\begin{array}{l}8.7 \mu \mathrm{m} \\
{[\mathrm{mJy}]}\end{array}$ & $\begin{array}{l}10.4 \mu \mathrm{m} \\
{[\mathrm{mJy}]}\end{array}$ & $\begin{array}{l}12.3 \mu \mathrm{m} \\
{[\mathrm{mJy}]}\end{array}$ & $\begin{array}{l}14.3 \mu \mathrm{m} \\
{[\mathrm{mJy}]}\end{array}$ \\
\hline $\mathrm{ChaH} \alpha 1$ & ${\mathrm{M} 7.5^{b}}^{2}$ & 2800 & 0.014 & $0.05^{b}$ & $2^{a, b}$ & $7 \pm 1$ & $6.7 \pm 1.8$ & $<11^{e}$ & - & $11.9 \pm 1.7$ & $11.9 \pm 0.7$ & $11.8 \pm 1.5$ \\
$2 \mathrm{M} 1207$ & $\mathrm{M}^{c}$ & 2600 & 0.0035 & 0.03 & $10^{a}$ & $7 \pm 1$ & - & $<8$ & $5.6 \pm 1$ & $7.5 \pm 1$ & - & - \\
\hline
\end{tabular}

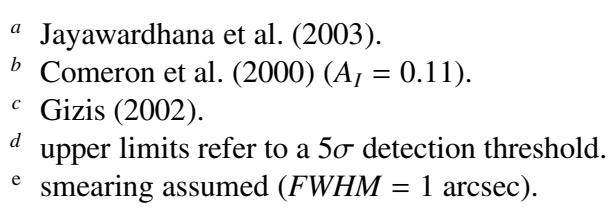

of circum(sub)stellar material, as it appears only marginally indicative (for ChaH $\alpha 1$ ) or even fails (for 2M1207).

\section{Disk models}

In this section we explain the main features of the SED using the new TReCS photometry (combined with ISO photometry, if available) with models of BD disks. The models are based on the Chiang \& Goldreich (1997) approximations, with modifications from Dullemond et al. (2001) for flux conservation. The general methodology and application to BD disks is explained in Pascucci et al. (2003). In view of the uncertainties of the exact shape of the SED over a wider wavelength range owing to the knowledge of only very few data points, we do not attempt to fine-tune the disk models by variations of input parameters like disk inner and outer radii, or inclination. We merely want to concentrate the discussion on those parameters that have the most notable impact on the shape of the SED around $10 \mu \mathrm{m}$. These are mainly the assumed disk geometry, and the dominant size and composition of the dust particle distribution. In the following we assume standard astronomical silicates with opacities from Draine \& Lee (1984). As we discuss only passive, re-processing disks, the assumed parameters of the BD mass and its luminosity are important. We directly use or derive them from published values as indicated.

\section{1. $\mathrm{ChaH} \alpha 1$}

According to Comeron et al. (2000), the spectral type of ChaH $\alpha 1$ is $\mathrm{M} 7.5$, and they estimate $T_{\text {eff }}=2770 \mathrm{~K}$ and $L=0.011 L_{\odot}$. Using the dusty BD model grids from Allard et al. $(2000,2001)$, we find that the available near-IR photometry for this object is in much better agreement with an atmospheric model for a $\approx 1 \mathrm{Myr}$ old BD with $T_{\text {eff }}=2800 \mathrm{~K}$ and $L=0.014 L_{\odot}$. We prefer these values for the BD photospheric parameters, which are anyway consistent with the Comeron et al. estimates, given their large errors. Figure 3 summarizes the results of our disk modeling in the MIR regime. The upper panel refers to opacities of astronomical (amorphous) silicates of $0.1 \mu \mathrm{m}$ size, while the lower panel displays the results of large, $2 \mu \mathrm{m}$ sized grains of the same composition. Full lines indicate the SED expected for a flared model, in which the vertical scale height is derived from local hydrostatical equilibrium at each disk radius. The dashed lines refer to the SED in a flat

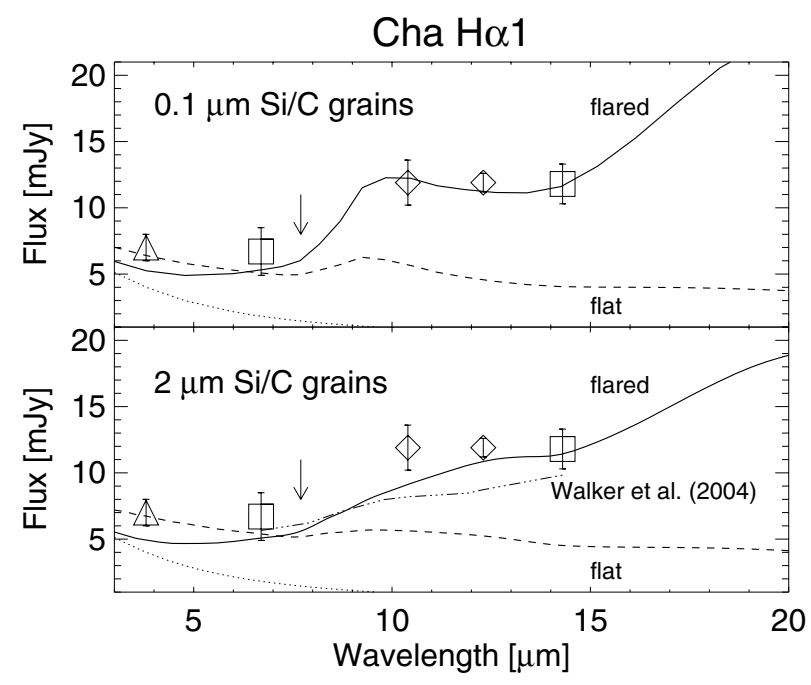

Fig. 3. SED and comparison of disk models for ChaH $\alpha 1$. Measurements are diamonds, with associated error bars. The upper panel refers to small dust sizes $(0.1 \mu \mathrm{m})$, the lower panel to larger grains $(2 \mu \mathrm{m})$. Flared (full line) and flat (dashed line) model predictions are compared. The contribution of the BD photosphere is approximated by a black-body model. The dashed-dotted line refers to the disk model by Walker et al. (2004). Our T-ReCS measurements are shown with diamonds, while the ISOCAM data are indicated with boxes and the $L^{\prime}$ point with a triangle, respectively.

geometrical configuration. Contributions from the photosphere are indicated as dotted lines. The dashed-dotted line indicates the results from Walker et al. (2004). We will refer to it later.

We note that the $L^{\prime}$ photometry from Jayawardhana et al. (2003) already indicates a significant excess, and favors - according to our modeling - a flattish disk geometry. However, the superb quality of our new TReCS measurements, especially at $10.4 \mu \mathrm{m}$, constrains the disk configuration to a standard flared disk model. Standard small ISM grains can explain the silicate emission feature very well, although larger grains cannot be excluded.

\subsection{MASS1207-3932}

Following Gizis (2002), the spectral type of 2M1207 is M8, which translates to $T_{\text {eff }}=2600 \mathrm{~K}$ according to the calibration of Luhman (1999). If we assume an age of $10 \mathrm{Myr}$ (see 


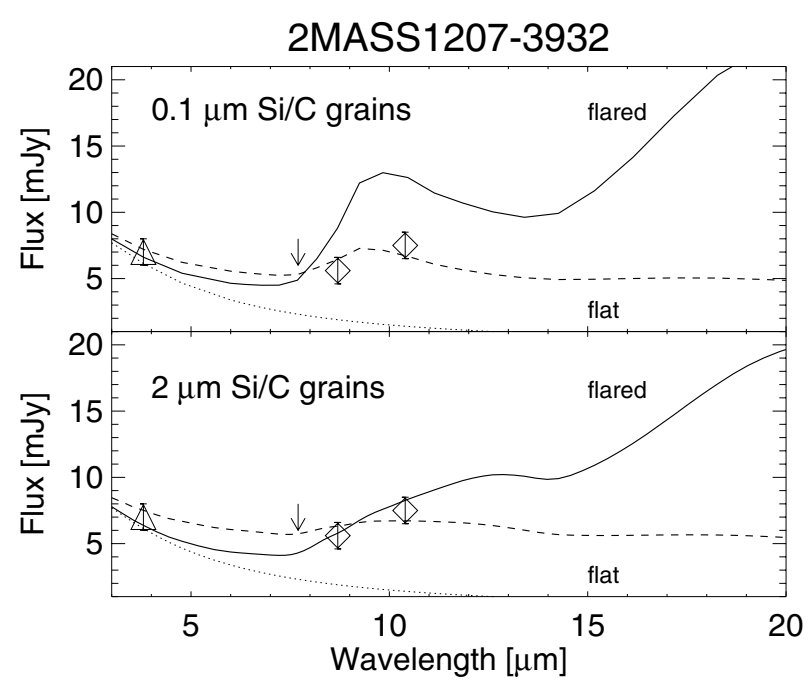

Fig. 4. SED and comparison of disk models for 2M1207.

Sect. 4.1), and scale the available $J H K$ photometry to a distance of $70 \mathrm{pc}$, we find an approximate bolometric luminosity of $0.0035 L_{\odot}$ and a mass of $0.003 M_{\odot}$ for this BD from the evolutionary tracks of Allard et al. (2000, 2001). These values are consistent with those estimated in MJBN.

The interpretation of the SED for this BD is not unique. ISO observations are not available, and the two TReCS measurements, together with the upper limit at $7.7 \mu \mathrm{m}$ and the $L^{\prime}$ measurement clearly exclude a standard flared disk model with ISM grain sizes $(0.1 \mu \mathrm{m})$. Either a flat disk or somewhat bigger grains $(2 \mu \mathrm{m})$ or both are compatible with the photometry. But large grains (up to $5 \mu \mathrm{m}$ ) only seem to be possible in a flared configuration viewed from a high inclination angle $(50 \mathrm{deg})$. The flat geometrical configuration is, on the other hand, more robust with respect to inclination angle variations, because shadowing effects are less pronounced.

Recently, Chauvin et al. (2004) report the detection of a giant planet candidate close to 2M1207. From near-IR spectroscopy they derive a spectral type of L5-L9.5, and a likely temperature of $T_{\text {eff }}=1250 \pm 200 \mathrm{~K}$ for this object, only 0.8 arcsec separated from the primary. Although it should be possible to spatially resolve it with an $8 \mathrm{~m}$ class telescope, we do not have any evidence for this object in any of our images (see Fig. 2). But we note that the expected photospheric flux in the $\mathrm{N}$-band is only around $0.2 \mathrm{mJy}$, assuming a simple black-body scaled to their $K$-band measurement. We therefore conclude that the excess flux we see is originating only from the disk material around 2M1207, and is not contaminated by the putative companion.

\section{Discussion}

Even with the new and improved MIR photometry an unambiguous interpretation remains difficult, because grain sizes and dust spatial distribution are coupled. For both objects, flared models can in principle explain the observed MIR fluxes. A flared disk containing small grains heated in the upper surface layers is the most likely explanation for the pronounced silicate feature in $\mathrm{ChaH} \alpha 1$. A flat geometry and substantial grain-growth are excluded for $\mathrm{ChaH} \alpha 1$. In the case of 2M1207 we find that a flared disk requires a large inclination and grains with sizes of a few $\mu \mathrm{m}$ to fit the two $10 \mu \mathrm{m}$ points. A flat disk composed of small, or moderately large grains up to $\approx 2 \mu \mathrm{m}$ can account for the observations equally well.

We will now try to put these observations in an evolutionary scenario.

\subsection{Grain growth and age evolution}

TWA membership of 2M1207 has been deduced both from proper motion (Gizis 2002), and radial velocities (MJBN). The age of the association itself is fairly well constrained: 8.3 Myrs from dynamical age determination (Makaraov \& Fabricius 2001), $\approx 10$ Myrs from isochrone fitting of its stellar population (Webb et al. 1999), and a similar age from Li abundance determination for late-type members (e.g. Soderblom et al. 1998). However, the presence of optically thick circumstellar disks in many of the prominent stellar members of TWA (TW-Hya, HD98800B, Hen3-600A, HR4796; see Jayawardhana et al. 1999) and signatures of active accretion like in the classical TTS TW-Hya (Rucinski \& Krautter 1983; Muzerolle et al. 2000) has always challenged a consistent evolutionary picture for this group of stars. Gap formation and significant dust evolution are observed in all four stellar systems mentioned above, and the detailed analysis of low-resolution N-band spectra makes it possible to decompose the $10 \mu \mathrm{m}$ feature into distinct dust species: TW Hya exhibits broad silicate emission, indicative for amorphous, large $(2 \mu \mathrm{m})$ olivine grains (Weinberger et al. 2002). HD 98800B has highly processed dust, dominated by large amorphous olivine and crystalline forsterite (Schütz et al. 2004). Also the spectrum of Hen3-600A features a rich mixture of crystalline silicate components, and even $\mathrm{SiO}_{2}$ (Honda et al. 2003).

In other words, all circumstellar disks in TWA show signs of dust processing, and are in particular incompatible with a primordial dust size distribution like in the ISM. Hence, the dust observed in these disks must have evolved on a $10 \mathrm{Myr}$ timescale since star formation. If the disk evolution is qualitatively similar around stars and sub-stellar objects, then one might expect similar dust properties also in circum-sub-stellar disks. The indication of larger grains in 2M1207 from our modeling is therefore fully consistent with this notion.

The case of ChaH $\alpha 1$ fits in this evolutionary scenario. Not much dust processing and/or growth has occurred, and one reason for this could well be its relatively young age ( 2 Myrs, or even <0.1 Myrs, Gomez \& Mardones 2003). Small grains are well-mixed throughout the vertical structure, which is itself in hydrostatical equilibrium, and illuminated by the central source.

\subsection{Comparison with other $B D s$}

Beside the two BDs discussed in this paper, the relation of disk geometry and grain size distribution has been investigated only in very few other BDs based on (ground-based) MIR photometry that sample the silicate feature well enough. 
Mohanty et al. (2004) discuss three BDs in the young $(<1 \mathrm{Myr}) \rho$-Oph star-forming region. For two of these objects (GY 310 and GY 11) they favor a standard, flared disk geometry, while a flat geometry cannot be ruled out for their third object (GY 5). Their data do not make it possible to draw strong constraints on the grain sizes, but small grains appear to fit the silicate feature better - at least in GY 310. In summary, these three very young BD do not show evidence for significant grain and disk evolution, consistent with the notion that grain growth occurs at later stages during disk evolution.

But this simple evolutionary picture might be more difficult to reconcile with the finding that other brown dwarf disks of similar age contain non-primordial dust, and larger grains than in the ISM. Good evidence for grain-growth is presented e.g. in Apai et al. (2004) for the $\approx 1$ Myr old brown dwarf BD Tau 4 . Although a precise age determination always suffers from notoriously uncertain assumptions about sub-stellar evolutionary tracks, and unknown distances, a much older age seems to be excluded because the object is already over-luminous for that particular isochrone (Martín et al. 2001). Nevertheless, this BD disk deviates from a flared disk geometry in equilibrium, and rather suggests that a significant amount of grain growth and dust settling has already occurred in its short lifetime. Also in the case of $\mathrm{ChaH} \alpha 2$, likely of similar age as $\mathrm{ChaH} \alpha 1$, only a flat disk geometry (Apai et al. 2002), and/or a somewhat larger grain size distribution (Walker et al. 2004) can explain the observed SED. Both possibilities are indicative for some dust/disk evolution that has not - yet - happened in $\mathrm{ChaH} \alpha 1$.

When, and how, does grain growth in circum(sub)stellar disks occur?

\subsection{Primordial grain growth}

There is some observational evidence that larger grains (and therefore opacities having a shallow wavelength dependence) are observable very early in disks around $\mathrm{T}$ Tauri stars (see e.g. Wood et al. 2002). It has been suggested that grain growth might therefore happen already during the collapse phase, or very early in the formation phase of the circumstellar disk (see, e.g. Suttner \& Yorke 2001). Based on this assumption, Walker et al. (2004) model the SED of BD disks, too, and find reasonable agreement with those $12 \mathrm{BD}$ disks that have ISOCAM MIR photometry. But not all of their model predictions are compatible with our observations. The Walker et al. (2004) model (indicated by a dot-dashed line in Fig. 3) fails, e.g., for $\mathrm{ChaH} \alpha 1$. Our new TReCS measurements rule out the possibility that the observed opacities (and therefore the grain sizes) deviate significantly from ISM properties. This means that at least for this objects grain growth and dust processing are not (yet) dominating the dust grain population as observed in the MIR.

\subsection{The role of multiplicity}

The co-existence of dust disks with vastly different grain properties has also been found in samples of classical TTS disks (Pryzgodda et al. 2003). Meeus et al. (2003), in particular, analyse the dust properties in a co-eval sample of TTS of similar (late) spectral type in Chamaeleon. A detailed mineralogical analysis of the N-band spectra of each individual object requires a different dust composition and size distribution, ranging from primordial ISM to highly processed dust. They note that the object that shows the highest degree of dust processing is in a close binary star, while the apparently unprocessed dust disk belongs to a single star. Without drawing strong conclusions they speculate that the presence of a binary might help dust processing in disks. We might be witnessing a similar trend in the observed BD disks in Chamaeleon. $\mathrm{ChaH} \alpha 2$ has been reported to be a close $\left(0.2^{\prime \prime}\right)$ binary (Neuhäuser et al. 2002), and its disk exhibits signs of significant dust processing and grain growth, in contrast to $\mathrm{ChaH} \alpha 1$, apparently a single BD (Joergens \& Guenther 2001).

There is no detailed model that explains the influence of a binary potential on the dynamics of a dust disk. But it seems plausible that a perturbing force acting on a circum-sub-stellar disk can induce enhanced collision, and therefore increase growth rates of dust particles in its disk (see, e.g. Dubrulle et al. 1995).

In this light, the overluminosity of BD Tau 4 in the HR diagram, together with its dust properties, might be a consequence of a close companion. Interestingly, Itoh et al. (1999) find a faint companion candidate near this object, though at rather wide separation $\left(\approx 4.2^{\prime \prime}\right)$, and its physical relation to BD Tau 4 is not established.

\section{Summary}

We report new high-quality MIR photometry from TReCS of two bona-fide brown dwarfs, and find excess emission in both cases, indicative of optical thick dust disks. We have investigated the influence of diverse disk geometries (flared versus flat) and of different grain size populations $(0.1 \mu \mathrm{m}$ versus $2 \mu \mathrm{m}$ ) on the MIR-SED of these brown dwarfs. In particular, we find:

- The MIR-SED of the $\approx 2 \mathrm{Myr}$ old ChaH $\alpha 1$ is fully consistent with a standard flared disk model, and primordial (ISM) grain size properties.

- The MIR-SED of the $\approx 10$ Myr old 2M1207 can be explained better by the presence of larger dust grains, and/or a flat disk geometry.

- The evolutionary timescale of dust in brown dwarfs appears to be similar to that in TTS.

- Similar to TTS, rapid grain growth and dust processing early in the BD disk evolution might be related to the presence of a close companion.

Acknowledgements. We thank C. Walker for providing her model fluxes of $\mathrm{ChaH} \alpha 1$ in electronic form. This research is based on observations obtained at the Gemini Observatory, which is operated by the Association of Universities for Research in Astronomy, Inc., under a cooperative agreement with the NSF on behalf of the Gemini partnership: the National Science Foundation (USA), the Particle Physics and Astronomy Research Council (UK), the National Research Council (Canada), CONICYT (Chile), the Australian Research Council 
(Australia), CNPq (Brazil), and CONICET (Argentina). We are indepted to the TRecS/Gemini teams for providing us with excellent data from service observations.

\section{References}

Allard, F., Hauschildt, P. H., Alexander, D. R., Tamanai, A., \& Ferguson, J. W. 2000, in From Giant Planets to Cool Stars, ed. C. A. Griffith, \& M. S. Marley (San Francisco: ASP), Conf. Ser. 212,127

Allard, F., Hauschildt, P. H., Alexander, D. R., Tamanai, A., \& Schweitzer, A. 2001, ApJ, 556, 357

Apai, D., Pascucci, I., Henning, T., et al. 2002, ApJ, 573, L115

Apai, D., Pascucci, I., Sterzik, M. F., et al. 2004, A\&A, 426, L53

Bontemps, S., André, P., Kaas, A. A., et al. 2001, A\&A, 359, 269

Bouwman, J., Meeus, G., de Koter, A., et al. 2001, A\&A, 375, 950

Chauvin, G., Lagrange, A.-M., Dumas., C., et al. 2004, A\&A, 425, L29

Chiang, E. I., \& Goldreich, P. 1997, ApJ, 490, 368

Chiang, E. I., Joung, M. K., Creech-Eakman, M. J., et al. 2001, ApJ, 547,1077

Comeron, F., Neuhäuser, R., \& Kaas, A. A. 2000, A\&A, 359, 269

Draine, B. T., \& Lee, H. M. 1984, ApJ, 285, 89

Dubrulle, B., Morfill, G., \& Sterzik, M. 1995, Icarus, 114, 237

Dullemond, C. P., Dominik, C., \& Natta, A. 2001, ApJ, 560, 957

Gomez, M., \& Mardones, D. 2003, AJ, 125, 2134

Gizis, J. E. 2002, ApJ, 575, 484

Honda, M., Kataza, H., Okamoto, Y. K., et al. 2003, ApJ, 585, L59

Itoh, Y., Tamura, M., \& Nakajima, T. 1999, AJ, 117, 1471

Jayawardhana, R., Hartmann, L., Fazio, G., et al. 1999, ApJ, 521, L129

Jayawardhana, R., Ardila, D. R., Stelzer, B., \& Haisch, K. E. Jr. 2003, AJ, 126, 1515

Joergens, V., \& Guenther, E. 2001, A\&A, 379, L9

Klein, R., Apai, D., Pascucci, I., Henning, T., \& Waters, L. B. F. M. 2003, ApJ, 593, L57

Liu, M. C., Najita, J., \& Tokunaga, A. T. 2003, ApJ, 585, 372
Luhman, K. L. 1999, ApJ, 525, L466

Makarov, V. V., \& Fabricius, C. 2001, A\&A, 368, 866

Martín, E., Dougados, C., Magnier E., et al. 2001, ApJ, 561, L195

Meeus, G., Waters, L. B. F. M., Bouwman, J., et al. 2001, A\&A, 365,476

Meeus, G., Sterzik, M. F., Bouwman, J., \& Natta, A. 2003, A\&A, 409, L25

Mohanty, S., Jayawardhana, R., \& Barrado y Navascues, D. 2003, ApJ, 593, L109 (MJBN)

Mohanty, S., Jayawardhana, R., Natta, A., et al. 2004, ApJ, 609, L33

Muench, A. A., Alves, J., Lada, C. J., \& Lada, E. A. 2001, ApJ, 558, L51

Muzerolle, J., Calvet, N., Briceno, C., Hartmann, L., \& Hillenbrand, L. 2000, ApJ, 535, L47

Neuhäuser, R., Brandner, W., Alves, J., Joergens, V., \& Cameron, F. 2002, A\&A, 384, 999

Natta, A., \& Testi, L. 2001, A\&A, 376, L22

Natta, A., Testi, L., Comeron, F., et al. 2002, A\&A, 393, 597

Pascucci, I., Apai, D., Henning, T., \& Dullemond, C.P. 2003, ApJ, 590, L111

Persi, P., Marenzi, A. R., Olofsson, G., et al. 2000, A\&A, 357, 219

Pryzgodda, F., van Boekel, R., Abraham, P., et al. 2003, A\&A, 412, L43

Rucinski, S. M., \& Krautter, J. 1983, A\&A, 121, 217

Schütz, O., Meeus, G., \& Sterzik, M. F. 2004, A\&A, in press

Suttner, G., \& Yorke, H. W. 2001, ApJ, 551, 461

Telesco, C. M., Pina, R. K., Hanna, K. T., et al. 1998, SPIE 3354, 534

van Boekel, R., Waters, L. B. F. M., Dominik, C., et al. 2003, A\&A, 400, L21

Walker, C., Wood, K., Lada, C. J., et al. 2004, MNRAS, in press

Webb, R. A., Zuckerman, B., Platais, I., et al. 1999, ApJ, 512, L63

Weinberger, A. J., Becklin, E. E., Schneider, G., et al. 2002, ApJ, 566,409

Wood, K., Wolff, M. J., Bjorkman, J. E., \& Whitney, B. 2002, ApJ, 564,887 